\title{
Efficacy of indoxacarb applied to cats against the adult cat flea, Ctenocephalides felis, flea eggs and adult flea emergence
}

Michael W Dryden ${ }^{1 *}$, Patricia A Payne ${ }^{1}$, Vicki Smith ${ }^{1}$, Kathleen Heaney ${ }^{2}$ and Fangshi Sun ${ }^{2}$

\begin{abstract}
Background: A study was conducted to evaluate the effect of indoxacarb applied to cats on adult cat fleas, Ctenocephalides felis, flea egg production and adult flea emergence.

Methods: Sixteen cats were selected for the study and allocated to two treatment groups. Eight cats were treated with a $19.5 \% \mathrm{w} / \mathrm{v}$ topical spot-on solution of indoxacarb on day 0 and eight cats served as untreated controls. Each cat was infested with 50 fleas on Days $-2,7,14,21,28,35$ and 42. On Days 1, 2, and 3, and at 2 and 3 days after each post treatment reinfestation flea eggs were collected from the pan under each cat cage. Eggs were counted and viability assessed by evaluating adult flea emergence 28 days after egg collection. Three days after treatment or infestation, each cat was combed to remove and count live fleas.

Results: Treatment with indoxacarb provided 100\% efficacy following infestations on day -2, 7, 14, 21 and 28 and efficacy was $99.6 \%$ following infestations on days 35 and 42 . Egg production from indoxacarb treated cats was reduced by $99.9 \%$ within 72 hours of treatment. For subsequent infestations no eggs were produced from treated cats from day 8 through day 30. Egg production was still reduced by $\geq 95.8 \%$ through day 45 . Indoxacarb treatment also reduced adult flea emergence from eggs for 5 weeks after treatment. The combination of reduction in egg numbers and egg viability from indoxacarb treated cats reduced predicted flea emergence by $100 \%$ from days 2 - 31 and $99.9 \%, 100 \%, 96.4 \%$ and $99.0 \%$ on days 37, 38, 44 and 45, respectively.

Conclusions: A topical spot-on formulation of indoxacarb provided $\geq 99.6 \%$ efficacy against flea infestations on cats for 6 weeks following a single treatment. Indoxacarb also eliminated or markedly reduced egg production for the entire evaluation period and reduced the viability of the few eggs that were produced from Day 1 through Day 38. Given indoxacarb's effect on adult fleas, egg production and egg viability; this formulation can interrupt flea reproduction on treated cats for at least 6 weeks after treatment.
\end{abstract}

Keywords: Flea, Ctenocephalides felis felis, Cat flea, Cats, Indoxacarb, Egg production, Adulticidal activity, Efficacy

\section{Background}

The cat flea, Ctenocephalides felis, is the most important ectoparasite of dogs and cats in most areas of the world [1]. Once cat fleas acquire a host, they mate and start laying eggs within $24-48$ hours [2,3]. Fleas deposit their eggs into the haircoat of the dog or cat with the eggs dropping off into the premises where within a few weeks they develop into adult fleas [3]. Since female cat

\footnotetext{
* Correspondence: Dryden@vet.k-state.edu

'Department of Diagnostic Medicine/Pathobiology, Kansas State University, Manhattan, KS 66506, USA

Full list of author information is available at the end of the article
}

fleas can produce 40 - 50 eggs/day it does not take long for massive numbers of flea eggs and ultimately emerging fleas to accumulate within the premises [3].

Currently, most flea control is based on residual topical spot-on treatments or oral medications [4-6]. The primary focus of these topical and systemic flea control efforts is to force fleas into "extinction" in a localized environment (home or yard) by preventing reproduction [6,7]. Eradication of an existing flea infestation is accomplished either by killing newly acquired fleas with a residual adulticide before they can initiate reproduction and/or affecting the viability of the eggs they produce

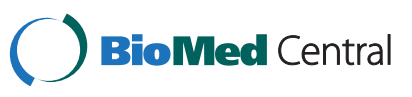


through the use of insect growth regulators or other ovicidal compounds [4]. In order for a topical or systemic insecticide to prevent egg production, the residual activity must be sufficient to kill or render moribund newly acquired fleas within 24 hours. A number of in-home and multicentric studies on three continents have demonstrated that this approach to flea control can be successful [6,8-13].

Indoxacarb is a novel oxadiazine insecticide that controls a broad spectrum of insects and was originally developed for use on a variety of plants including vegetables, fruit trees, cotton and row crops [14]. Indoxacarb is a pro-insecticide that is metabolized within the insect to a more active form: an $\mathrm{N}$-decarbomethoxylated metabolite of indoxacarb. This metabolic conversion of indoxacarb, known as bioactivation, is attributed to actions of esterase and amidase enzymes within the insect $[15,16]$. The active metabolite of indoxacarb exerts its effect by blocking the voltage-gated sodium ion channels in insects and is at least forty times more potent than parent indoxacarb in its ability to block sodium channel ion current [15-17]. Mammals exhibit minimal bioactivation of indoxacarb and sodium channel binding studies in rats show the active indoxacarb metabolite has much weaker potency against mammalian sodium channels than insect channels $[16,18]$.

This study was undertaken to evaluate the efficacy of a recently developed spot-on formulation of indoxacarb applied to cats against adult fleas, flea egg production and development of fleas from eggs to adults.

\section{Methods}

\section{Animals and housing}

Nineteen (10m:9f; > 6mo; 2.7 to $4.2 \mathrm{~kg}$ ), purpose bred domestic shorthair cats were used in this study. Cats used in this investigation had no exposure to ectoparasiticides prior to treatment and were in good health throughout the study. Cats were housed individually in standard stainless cages steel with solid sides and back, with a steel barred door. Cats were physically separated by the solid stainless steel cage walls. Cats were fed a commercial dry cat-food ration. Water was available ad libitum. Physical examinations were conducted by a licensed veterinarian prior to the initial flea infestation and cats were determined to be in good health and free of any preexisiting dermal lesions. All animal care procedures conformed to guidelines established by the Institutional Animal Care and Use Committee at Kansas State University (Approval No. 3124).

\section{Animal selection and randomization}

On Day -8 , all cats were infested with 50 adult cat fleas, C. felis, (KS1 strain) 1 to 5 days post emergence. On Day -5 , flea comb counts were performed to evaluate the susceptibility of each cat to maintain experimental infestations and for random allocation of the cats to the treatment groups. Cats were combed with a fine-toothed flea comb having 12 to 13 teeth $/ \mathrm{cm}$. Flea removal was achieved by combing each cat thoroughly for $10 \mathrm{mi}-$ nutes. If five or more fleas were recovered during this period, the cat was combed for an additional 5 minutes. If any fleas were recovered during the second combing period, the cats were combed for an additional 5 minutes. Personnel conducting flea combing and flea counts were blinded to treatment groups. The 16 cats $(7 \mathrm{~m}: 9 \mathrm{f})$ with the highest pre-treatment flea counts were used for the study. Within gender, cats were ranked from highest to lowest flea count, with the female cat with lowest flea number placed in male gender grouping, and blocked in two groups containing 8 cats each.

\section{Treatments}

Cats $(4 \mathrm{~m}: 4 \mathrm{f})$ in treatment group 1 were treated topically with indoxacarb $(2-4.0 \mathrm{~kg}$ were treated with $0.51 \mathrm{~mL}$ and cats $>4.1 \mathrm{~kg}$ were treated with $1.03 \mathrm{~mL}$ indoxacarb, 19.5\% w/v; ACTIVYL ${ }^{\circ}$ Merck Animal Health) applied using the commercial product pipette or a syringe. Treatment was administered by parting the hair with the tip of the pipette or syringe at the base of the skull of the cat and the entire contents were applied directly to the skin. Cats (3m:5f) in treatment group 2 served as untreated controls. Cats were observed following treatment for any adverse events associated the treatments.

\section{Efficacy evaluations}

On Days -2, 7, 14, 21, 28, 35, and 42, each cat was infested with approximately 50 unfed cat fleas, C. felis (KS1 strain). On Days 1, 2, and 3, and at 2 and 3 days after each post treatment reinfestation flea eggs were collected from the pan under each cat cage. Prior to the egg collections, cats were massaged/brushed vigorously by hand for $\sim 20$ seconds in their cages to dislodge any flea eggs from the cat's hair coat, allowing the eggs to fall into the drop pan below the cage. Eggs collected were counted by placing the eggs in a glass Petri dish and counted under a dissecting microscope. At approximately 72 hours after treatment or infestation, each cat was combed to remove and count live fleas. Viability of eggs was determined by placing up to 100 eggs into glass Petri dishes containing flea growth media and holding in a rearing chamber (I-30B, Percival Manufacturing Co., Boone, IA) at $27-28^{\circ} \mathrm{C}, 70-80 \%$ relative humidity, 24 hours dark. If less than 100 eggs were collected, the entire sample was placed into the Petri dish containing flea growth media and similarly processed. At $10-12$ days after egg collection, pupae (and any larvae that had not completed cocoon formation) were sifted from the media and placed into plastic vials with lids. Adult emergence 
was determined by counting adult fleas at 28 days after egg collection. Personnel conducting comb counts, egg collections and viability assessments were blinded to treatment group allocation.

\section{Data analysis}

Data at each time point were analyzed separately. The adult flea and egg count data were transformed prior to analysis using the $\mathrm{Y}=\log _{\mathrm{e}}(\mathrm{x}+1)$ transformation. The $\log$ transformed data were analyzed by a mixed linear model including treatment as the fixed effect; and block as the random effect. Least squares means were used for treatment comparisons and were back transformed to obtain the estimates of geometric mean flea and egg counts. Percentages of the emerging adult flea count were analyzed by a mixed linear model including treatment as the fixed effect; and block as the random effect. A twotailed test was used for the comparison between groups. Statistical significance was declared when $\mathrm{p}<0.05$. The primary software was SAS version 9.3.

Percent control of adult fleas was calculated using geometric means with Abbott's formula:

$$
\operatorname{Efficacy}(\%)=100 \times(\mathrm{MC}-\mathrm{MT}) / \mathrm{MC}
$$

Where:

- MC is the geometric mean number of total adult live fleas on untreated cats

- MT is the geometric mean number of total adult live fleas on treated cats.

The percent control of egg production and egg viability were also calculated using Abbott's formula by adjusting the mean number of eggs produced and mean percent of emergent fleas from treated cats with the mean number of eggs produced and mean percent of emergent fleas produced by fleas on untreated control cats.

\section{Results}

All cats included in the study demonstrated adequate pretreatment flea retention with day -5 flea counts ranging from 16 - 38 fleas/cat. Nontreated cats also maintained adequate flea infestations throughout the study with geometric mean flea counts ranging from 19.3 to 28.0 (Table 1).

Cats in the indoxacarb treatment group were administered an average of $33.7 \mathrm{mg} / \mathrm{kg}(27.3-47.8 \mathrm{mg} / \mathrm{kg})$ indoxacarb. This single dose of indoxacarb produced highly significant reductions in geometric mean flea counts on treated cats throughout the entire 45 days of the study $(\mathrm{P}<0.001$, Table 1$)$. Treatment with indoxacarb provided $100 \%$ efficacy following infestations on day $-2,7,14$, 21 and 28 and $99.6 \%$ reduction in geometric mean
Table 1 Geometric mean adult flea counts and percent efficacy relative to non-treated controls for cats treated with a $19.5 \% \mathrm{w} / \mathrm{v}$ indoxacarb topical spot-on

\begin{tabular}{ccc}
\hline Study day & \multicolumn{2}{c}{ Mean \# of Fleas $^{\mathbf{1 , 2}}$} \\
\cline { 2 - 3 } & Non-treated controls $^{\mathbf{3}}$ & Indoxacarb $^{\mathbf{3}}$ \\
\hline 3 & $27.2^{\mathrm{a}}$ & $0.0^{\mathrm{b}}(100.0)^{4}$ \\
10 & $19.3^{\mathrm{a}}$ & $0.0^{\mathrm{b}}(100.0)$ \\
17 & $25.6^{\mathrm{a}}$ & $0.0^{\mathrm{b}}(100.0)$ \\
24 & $28.0^{\mathrm{a}}$ & $0.0^{\mathrm{b}}(100.0)$ \\
31 & $24.7^{\mathrm{a}}$ & $0.0^{\mathrm{b}}(100.0)$ \\
38 & $25.7^{\mathrm{a}}$ & $0.1^{\mathrm{b}}(99.6)$ \\
45 & $20.8^{\mathrm{a}}$ & $0.1^{\mathrm{b}}(99.6)$ \\
\hline
\end{tabular}

${ }^{1}$ Each cat was infested with 50 adult Ctenocephalides felis from the KS1 strain on days $-2,7,14,21,28,35 \& 42$.

${ }^{2}$ Geometric mean \# of fleas recovered from cats per treatment group.

${ }^{3}$ Each of 8 cats in the control group received no treatment. Each of 8 cats in the $19.5 \% \mathrm{w} / \mathrm{v}$ indoxacarb group were administered the topical spot-on according to label directions on Day 0.

${ }^{4} \%$ efficacy $=$ ( (geometric mean live adult flea count control-geometric mean live adult flea count treatment)/geometric mean live adult flea count control) $\times 100$.

$\mathrm{a}, \mathrm{b}$ geometric means across rows with unlike letter superscripts are significantly different $(\mathrm{P}<0.001)$.

Table 2 Geometric mean flea egg counts and percent efficacy relative to non-treated controls for cats treated with a $19.5 \% \mathrm{w} / \mathrm{v}$ indoxacarb topical spot-on

\begin{tabular}{|c|c|c|c|}
\hline \multirow[b]{2}{*}{$\begin{array}{l}\text { Day of egg } \\
\text { collection }\end{array}$} & \multirow{2}{*}{$\frac{\text { Non-treated controls }^{1,2}}{\text { Mean \# of eggs }}$} & \multicolumn{2}{|c|}{ Indoxacarb ${ }^{1,2}$} \\
\hline & & Mean \# of eggs & Efficacy $^{4}$ \\
\hline 1 & $654.2^{a}$ & $220.9^{a}$ & 66.2 \\
\hline 2 & $627.8^{a}$ & $7.2^{b}$ & 98.9 \\
\hline 3 & $589.8^{a}$ & $0.6^{b}$ & 99.9 \\
\hline 9 & $650.7^{a}$ & $0.0^{b}$ & 100.0 \\
\hline 10 & $957.6^{a}$ & $0.0^{b}$ & 100.0 \\
\hline 16 & $459.1^{a}$ & $0.0^{b}$ & 100.0 \\
\hline 17 & $686.4^{a}$ & $0.0^{b}$ & 100.0 \\
\hline 23 & $739.8^{a}$ & $0.0^{b}$ & 100.0 \\
\hline 24 & $1086.1^{a}$ & $0.0^{b}$ & 100.0 \\
\hline 30 & $566.5^{a}$ & $0.0^{b}$ & 100.0 \\
\hline 31 & $732.1^{a}$ & $0.1^{b}$ & 99.9 \\
\hline 37 & $491.2^{a}$ & $0.7^{b}$ & 99.9 \\
\hline 38 & $577.9^{a}$ & $0.1^{b}$ & 99.9 \\
\hline 44 & $229.7^{a}$ & $9.7^{b}$ & 95.8 \\
\hline 45 & $508.4^{a}$ & $6.8^{b}$ & 98.7 \\
\hline
\end{tabular}

${ }^{1}$ Each of 8 cats in the control group received no treatment. Each of 8 cats in the $19.5 \% \mathrm{w} / \mathrm{v}$ indoxacarb group were administered the topical spot-on according to label directions on Day 0.

${ }^{2}$ Each cat was infested with 50 adult Ctenocephalides felis from the KS1 strain on days $-2,7,14,21,28,35 \& 42$.

${ }^{3}$ Geometric mean \# of eggs recovered from cats per treatment group.

${ }^{4} \%$ efficacy $=$ ((geometric mean \# eggs control-geometric mean \# eggs

treatment)/geometric mean \# eggs control) $\times 100$.

$\mathrm{a}, \mathrm{b}$ Geometric means across rows with unlike letter superscripts are significantly different $(P<0.001)$. 
flea counts following infestations on days 35 and 42 (Table 1).

Geometric mean flea egg counts for the indoxacarbtreated cats were also significantly lower than those for nontreated controls at all post-treatment evaluations $(\mathrm{P}<0.001$, Table 2$)$. Egg production from indoxacarb treated cats was reduced by $66.2 \%$ within 24 hours after treatment and $99.9 \%$ within 72 hours of treatment. For subsequent infestations egg production was non-existent from treated cats out to day 30 and $99.9 \%$ on day 31 relative to nontreated cats (Table 2). Egg production was still markedly reduced by $95.8 \%$ and $98.7 \%$ on days 44 and 45 , respectively. The geometric mean number of eggs produced from fleas on control cats from day 9 through day 45 post-treatment ranged from 229.7 1,086.1. Whereas, the geometric mean number of eggs from indoxacarb treated cats was highest on day 44 at 9.7 eggs/cat (Table 2).

Based on percent adult emergence, indoxacarb treatment also had an apparent lethal effect on the viability of flea eggs collected from treated cats on days 1-3 and on days 31 and $38(\mathrm{P}<0.001$, Table 3$)$. Since no eggs were collected from indoxacarb treated cats from days $9-30$ no percent adult flea emergence analysis could be conducted. A direct effect on egg viability based on percent adult emergence was not observed on post-treatment days 44 and 45 . However when egg viability is considered along with the marked reduction in egg numbers, indoxacarb did demonstrate a marked effect upon the reproductive success of the fleas even at post-treatment day 45 (Table 3). The combination of the geometric mean number of eggs produced and the percent adult emergence from those eggs results in the predicted adult emergence or reproductive success. The predicted adult emergence from eggs produced by fleas on the untreated control cats ranged from 177.5 - 908.2 emerging fleas/cat. Whereas the reduction in predicted flea emergence from indoxacarb treated cats was $100 \%$ from days $2-31$ and was still $96.8 \%$ and $99.0 \%$ on days 44 and 45 , respectively (Table 3).

There were no adverse events associated with indoxacarb treatments in this study.

\section{Discussion}

Veterinarians and pet owners should expect that when correctly applied indoxacarb spot-on will control the existing flea burden on a cat and that there should be a rapid reduction in egg production from the existing flea

Table 3 Geometric mean percent adult emergence, predicted adult emergence and percent efficacy relative to non-treated controls for predicted adult flea emergence from eggs collected from cats treated with a $19.5 \% \mathrm{w} / \mathrm{v}$ indoxacarb topical spot-on

\begin{tabular}{|c|c|c|c|c|c|}
\hline \multirow[b]{2}{*}{ Study day ${ }^{3}$} & \multicolumn{2}{|c|}{ Non-treated controls ${ }^{1,2}$} & \multicolumn{3}{|c|}{ Indoxacarb $^{1,2}$} \\
\hline & $\%$ Adult emergence ${ }^{4}$ & Predicted emergence $^{5}$ & $\%$ Adult emergence $^{4}$ & Predicted emergence ${ }^{5}$ & Efficacy $^{6}$ \\
\hline 1 & $50.1^{\mathrm{a}}$ & 327.9 & $4.6^{b}$ & 10.2 & 96.9 \\
\hline 2 & $46.1^{\mathrm{a}}$ & 289.6 & $0.0^{\mathrm{b}}$ & 0.0 & 100.0 \\
\hline 3 & $67.0^{\mathrm{a}}$ & 395.2 & $0.0^{\mathrm{b}}$ & 0.0 & 100.0 \\
\hline 9 & 77.1 & 501.8 & $\mathrm{NE}$ & 0.0 & 100.0 \\
\hline 10 & 79.8 & 763.7 & $\mathrm{NE}$ & 0.0 & 100.0 \\
\hline 16 & 75.3 & 345.5 & NE & 0.0 & 100.0 \\
\hline 17 & 76.4 & 524.2 & $\mathrm{NE}$ & 0.0 & 100.0 \\
\hline 23 & 84.3 & 623.2 & NE & 0.0 & 100.0 \\
\hline 24 & 83.6 & 908.2 & $\mathrm{NE}$ & 0.0 & 100.0 \\
\hline 30 & 77.0 & 436.2 & $\mathrm{NE}$ & 0.0 & 100.0 \\
\hline 31 & $85.2^{\mathrm{a}}$ & 604.0 & $0.0^{\mathrm{b}}$ & 0.0 & 100.0 \\
\hline 37 & $75.4^{\mathrm{a}}$ & 370.2 & $20.0^{\mathrm{b}}$ & 0.1 & 99.9 \\
\hline 38 & $63.7^{\mathrm{a}}$ & 388.6 & $0.0^{\mathrm{b}}$ & 0.0 & 100.0 \\
\hline 44 & $77.3^{\mathrm{a}}$ & 177.5 & $66.1^{\mathrm{a}}$ & 6.4 & 96.4 \\
\hline 45 & $82.3^{\mathrm{a}}$ & 418.1 & $62.5^{\mathrm{a}}$ & 4.3 & 99.0 \\
\hline
\end{tabular}

${ }^{1}$ Each of 8 cats in the control group received no treatment. Each of 8 cats in the $19.5 \% \mathrm{w} / \mathrm{v}$ indoxacarb group were administered the topical spot-on according to label directions on Day 0.

${ }^{2}$ Each cat was infested with 50 adult Ctenocephalides felis from the KS1 strain on days $-2,7,14,21,28,35$ \& 42.

${ }^{3}$ Days post-treatment that eggs were collected.

$4 \%$ Adult emergence $=(\#$ of adults emerging/\# of eggs collected $) \times 100$.

${ }^{5}$ Predicted emergence $=(\%$ emergence $\times$ geometric mean \# eggs collected from Table 2$)$.

$6 \%$ Efficacy $=($ (predicted adult emergence control-predicted adult emergence treatment $) /$ geometric mean \% adult emergence control $) \times 100$.

$\mathrm{NE}=$ no flea eggs collected.

a,b Values across rows with unlike superscripts are significantly different $(P<0.05)$. 
infestation. Within 48 hours of application egg production was almost completely halted ( $98.9 \%$ reduction). Since none of those eggs produced adult fleas no dissemination of viable eggs occurred from treated cats within 2 days of treatment.

Most currently available residual topical spot-on and systemic insecticide formulations are marketed to provide at least 30 days of effective flea control. Indoxacarb spot-on certainly provided and exceeded that level of control. A single application of indoxacarb provided $\geq 99.6 \%$ control of adult cat fleas on cats for at least 45 days after treatment.

Following treatment, egg production from weekly flea reinfestations was reduced by $\geq 99.9 \%$ at least 5 weeks. Consumption of blood is necessary before cat fleas can initiate reproduction and egg production does not begin until 24-48 hours after females take their first blood meal [1,2]. Therefore, if a residual insecticide can kill or produce toxicity in newly acquired fleas within 24 hours, egg production should be markedly reduced or halted. While this study did not evaluate the residual speed of kill of indoxacarb or reduction of blood consumption by adult fleas, it did demonstrate that indoxacarb had a profound effect upon egg production. This indirectly indicates that indoxacarb is rapidly toxic to fleas and thus markedly reduces blood feeding by fleas.

Indoxacarb also had an effect upon adult flea emergence from eggs. While this study did not evaluate egg hatch directly it is anticipated that the effect of indoxacarb was on egg viability. Eggs collected from trays under treated cats were sorted and removed from all debris from those cats. The eggs were subsequently placed into standard rearing media. Following this procedure any larvae hatching from those eggs would have had no further contact with indoxacarb. Similar marked lethal effect on egg viability by a flea adulticide has been previously observed with selamectin [19].

Effective residual flea adulticides should have sufficient residual activity to provide for continuing kill of most if not all the newly emerging fleas that jump onto the treated pet between applications. If a flea product also has an effect upon the viability of the eggs produced by any female flea surviving the adulticidal effect, that product would markedly suppress the reproductive success of a flea population [6,7]. In this study indoxacarb demonstrated marked flea kill, profound reduction in egg production and apparent ovicidal activity, as has been observed in other insect species [15]. This combination of attributes provided $\geq 99.9 \%$ reduction in reproductive success (predicted emergence) for 5 weeks following a single application of indoxacarb. Proper application of indoxacarb to cats in a flea infested home should effectively drive the flea population to extinction.
This study was conducted using the KS1 flea strain. Several previous studies have demonstrated that this strain has reduced susceptibility or outright resistance to carbaryl, chlorpyriphos, fenthion, fipronil, imidacloprid, permethrin, pyrethrins, and spinosad [3,19-24]. This study showed that indoxacarb performed well against this KS-1 flea strain.

\section{Conclusions}

A topical spot-on formulation of indoxacarb provided $100 \%$ efficacy against flea infestations on cats for 4 weeks following a single treatment and was still providing 99.6\% reduction in flea counts through week 6 . Indoxacarb eliminated or markedly reduced egg production for the entire evaluation period. The formulation also reduced the viability of the few eggs that were produced through Day 38. Given indoxacarb's effect on adult fleas, egg production and egg viability; it appears this formulation can interrupt flea reproduction for at least 6 weeks after treatment.

\section{Competing interests}

MWD has had research funded and has been sponsored to lecture by numerous pharmaceutical companies, including Merck Animal Health. Kathleen Heaney and Fangshi Sun are employees of Merck Animal Health.

\section{Authors' contributions}

MWD assisted in the design of the study, served as study investigator and drafted the manuscript. VS \& PAP coordinated and supervised data collection and entry and revision of manuscript; $\mathrm{KH}$ assisted in design of study, monitoring of study and manuscript revision. FS conducted the statistical analysis of the data. All authors reviewed and approved the final manuscript.

\section{Acknowledgements}

This study was funded in part by and publications fees paid by Merck Animal Health

\section{Author details}

${ }^{1}$ Department of Diagnostic Medicine/Pathobiology, Kansas State University, Manhattan, KS 66506, USA. ${ }^{2}$ Merck Animal Health, 556 Morris Avenue, Summit, NJ 07901, USA.

Received: 5 April 2013 Accepted: 26 April 2013

Published: 3 May 2013

\section{References}

1. Blagburn BL, Dryden MW: Biology, treatment and control of flea and tick infestations. Vet Clin N Am 2009, 39(6):1173-1200.

2. Akin DE: Relationship between feeding and reproduction in the cat flea, Ctenocephalides felis (Bouche). M.S. Thesis: University of Florida, Department of Entomology; 1984.

3. Dryden MW: Host association, on host longevity and Egg production of ctenocephalides felis. Vet Parasitol 1989, 34:117-122.

4. Dryden MW, Broce AB: Integrated flea control for the $21^{\text {st }}$ Century. Comp Cont Ed Pract Vet 2002, 24((1) suppl):36-39.

5. Rust MK: Advances in the control of Ctenocephalides felis felis (cat flea) on cats and dogs. Trends Parasitol 2005, 21:232-236.

6. Chin A, Lunn P, Dryden MW: Persistent flea infestations in dogs and cats controlled with monthly topical applications of fipronil and methoprene. Aust Vet Pract 2005, 35(3):89-96.

7. Dryden MW: Flea and tick control in the $21^{\text {st }}$ century, challenges and opportunities. Vet Dermatol 2009, 20:435-440.

8. Dryden MW, Perez HR, Ulitchny DM: Control of flea populations on naturally infested dogs and cats and in private residences with either 
topical imidacloprid spot application or the combination of oral lufenuron and pyrethrin spray. Am J Vet Med Assoc 1999, 215(1):36-39.

9. Dryden MM, Magid-Denenberg T, Bunch S: Control of fleas on naturally infested dogs and cats and in private residences with topical spot applications of fipronil or imidacloprid. Vet Parasitol 2000, 93:69-75.

10. Robertson-Plouch C, Baker KA, Hozak RR, Zimmermann AG, Parks SC, Herr C, Hart LM, Jay J, Hutchens DE, Snyder DE: Clinical field study of the safety and efficacy of spinosad chewable tablets for controlling fleas on dogs. Vet Therapeutics 2008, 9:26-36.

11. Beugnet $F$, Franc M: Results of a European multicentric field efficacy study of fipronil-(S) methoprene combination on flea infestation of dogs and cats during 2009 summer. Parasite 2010, 17(4):337-342.

12. Dryden MW, Payne PA, Smith V, Riggs B, Davenport J, Kobuszewski D: Efficacy of dinotefuran-pyriproxyfen, dinotefuran-pyriproxyfenpermethrin and fipronil-(S)-methoprenetopical spot-on formulations to control flea populations in naturally infested pets and private residences in Tampa, FL. Vet Parasitol 2011, 182:281-286.

13. Dryden MW, Ryan WG, Bell M, Rumschlag AJ, Young LM, Snyder DE: Assessment of owner-administered monthly treatments with oral spinosad or topical spot-on fipronil/(S)-methoprene in controlling fleas and associated pruritus in dogs. Vet Parasitol 2013, 191:340-346.

14. McCann SF, Cordova D, Andaloro JT, Lahm GP: Sodium channel blocking insecticides, indoxacarb. In Modern crop protection compounds. Volume 1 . Edited by Kramer W, Schirmer U. Wenheim: Wiley-VCH; 2007:1031-1048.

15. Wing KD, Andaloro JT, McCann SF, Salgado VL: Indoxacarb and the sodium channel blocker insecticides: chemistry, physiology and biology in insects. In Insect control biological and synthetic agents. Edited by Gilbert LI, Gill SS. London: Elsevier, B.V; 2010:35-57.

16. Wing KD, Sacher M, Kagaya Y, Tsurubuchi Y, Mulderig L, Connair M, Schnee M: Bioactivation and mode of action of the oxadiazine indoxacarb in insects. Crop Prot 2000, 19:537-545.

17. Silver KS, Song W, Nomura Y, Salgado VL, Dong K: Mechanism of action of sodium channel blocker insecticides (SCBIs) on insect sodium channels. Pest Biochem Phys 2010, 97:87-92.

18. Zhao X, Ikeda T, Yeh TZ, Narahashi T: Voltage-dependent block of sodium channels in mammalian neurons by the oxadiazine insecticide indoxacarb and its metabolite DCJW. NeuroToxicol 2003, 24:83-96.

19. Dryden M, Payne P, Smith V: Efficacy of selamectin and fipronil / (S)-methoprene spot-on formulations applied to dogs against the adult cat flea, Ctenocephalides felis, flea eggs and adult flea emergence. Vet Therapeutics 2007, 8:255-262.

20. Payne PA, Dryden MW, Smith V, Ridley RK: Effect of $0.29 \% \mathrm{w} / \mathrm{w}$ fipronil spray on adult flea mortality and egg production of three different cat flea, Ctenocephalides felis (Bouché), strains infesting dogs. Vet Parasitol 2001, 102(4):331-340.

21. Bossard RL, Dryden MW, Broce AB: Insecticide susceptibilities of cat fleas (Siphonaptera: Pulicidae) from several regions of the United States. J Med Entomol 2002, 39:742-746.

22. Rust MK, Waggoner M, Hinkle NC, Mencke N, Hansen O, Vaughn M, Dryden MW, Payne P, Blagburn B, Jacobs DE, Bach T, Bledsoe D, Hopkins T, Mehlhorn $\mathrm{H}$ : Development of a larval bioassay for susceptibility of cat fleas (Siphonaptera: Pulicidae) to imidacloprid. J Med Entomol 2002, 39:671-674.

23. Bass C, Schroeder I, Turberg A, Field L, Williamson MS: Identification of mutations associated with pyrethroid resistance in the para-type sodium channel of the cat flea, Ctenocephalides felis. Insect Biochem Mol Bio 2004, 34:1305-1313.

24. Dryden MW, Payne PA, Smith V, Kobuszewski D: Efficacy of topically applied dinotefuran formulations and orally administered spinosad tablets against the KS1 flea strain infesting dogs. Intern J Appl Research Vet Med 2010, 9(2):123-128.

doi:10.1186/1756-3305-6-126

Cite this article as: Dryden et al: Efficacy of indoxacarb applied to cats against the adult cat flea, Ctenocephalides felis, flea eggs and adult flea emergence. Parasites \& Vectors 2013 6:126.

\section{Submit your next manuscript to BioMed Central and take full advantage of:}

- Convenient online submission

- Thorough peer review

- No space constraints or color figure charges

- Immediate publication on acceptance

- Inclusion in PubMed, CAS, Scopus and Google Scholar

- Research which is freely available for redistribution

Submit your manuscript at www.biomedcentral.com/submit
C Biomed Central 